\title{
Eicosanoid content in fetal calf serum accounts for reproducibility challenges in cell culture
}

Laura Niederstaetter ${ }^{1} \ddagger$, Benjamin Neuditschko ${ }^{1,2} \ddagger$, Julia Brunmair ${ }^{1}$, Lukas Janker ${ }^{1}$, Andrea Bileck ${ }^{1,3}$, Giorgia Del Favero ${ }^{4,5}$, Christopher Gerner ${ }^{1,3}$

${ }^{1}$ Department of Analytical Chemistry, Faculty of Chemistry, University of Vienna

${ }^{2}$ Department of Inorganic Chemistry, Faculty of Chemistry, University of Vienna

${ }^{3}$ Joint Metabolome Facility, Faculty of Chemistry, University of Vienna

${ }^{4}$ Department of Food Chemistry and Toxicology, Faculty of Chemistry, University of Vienna

${ }^{5}$ Core Facility Multimodal Imaging, Faculty of Chemistry, University of Vienna

¥Authors contributed equally to the work 
bioRxiv preprint doi: https://doi.org/10.1101/2020.09.18.303313; this version posted September 18, 2020. The copyright holder for this preprint (which was not certified by peer review) is the author/funder, who has granted bioRxiv a license to display the preprint in perpetuity. It is made available under aCC-BY-ND 4.0 International license.

\section{Abbreviations}

FFA - Free fatty acids, CDM - Chemically defined medium, SA - Serum Albumin, PUFA Polyunsaturated fatty acids, FBS - Fetal bovine serum, FCS - Fetal calf serum, PMA - Phorbol 12myristate 13-acetate, LPS - Lipopolysaccharides, AA - Arachidonic acid, HETE Hydroxyeicosatetraenoic Acid, 


\begin{abstract}
Reproducibility issues regarding in vitro cell culture experiments are related to genetic fluctuations and batch-wise variations of biological materials such as fetal calf serum (FCS). Genome sequencing may control the former, while the latter may remain unrecognized. Using a U937 macrophage model for cell differentiation and inflammation, we investigated whether the formation of effector molecules was dependent on the FCS batch used for cultivation. High resolution mass spectrometry was used to identify FCS constituents and to explore their effects on cultured cells evaluating secreted cytokines, eicosanoids and other inflammatory mediators. Remarkably, the FCS eicosanoid composition showed more batch-dependent variations than the protein composition. Efficient uptake of fatty acids from medium by U937 macrophages and inflammation-induced release thereof was evidenced using C13-labelled arachidonic acid, highlighting rapid lipid metabolism. For functional testing, FCS batch-dependent nanomolar concentration differences of two selected eicosanoids, 5-HETE and 15-HETE, were balanced out by spiking in. Culturing U937 cells at these defined conditions indeed resulted in significant proteome alterations indicating HETE-induced PPAR $\gamma$ activation, independently corroborated by HETE-induced formation of peroxisomes observed by high-resolution microscopy. In conclusion, the present data demonstrate that FCS-contained eicosanoids, subject to substantial batch-wise variation, may modulate cellular effector functions in cell culture experiments.
\end{abstract}




\section{Introduction}

Problems with the inter-laboratory reproducibility of results obtained with in vitro cell culture models are increasingly recognized ${ }^{1,2}$. Actually, the need to reduce the use of animal models for research purposes relies also on the use of accurate in vitro test models ${ }^{3,4}$. Important decisions such as the choice of drug candidates to be evaluated in clinical studies may be based on such experiments ${ }^{5}$. Thus, the identification of influencing factors potentially modulating such in vitro data is mandatory. Biological materials and reference materials have been recognized as main contributors for irreproducibility ${ }^{5}$, resulting in a current focus on the investigation of genetic heterogeneity and genetic instability of cell culture models ${ }^{6,7}$. Actually, here we are presenting Fetal Bovine Serum (FBS; also fetal calf serum, FCS) as another relevant contributor to reproducibility issues. FCS is commonly used as cell culture supplement sustaining the growth and duplication of mammalian cells in vitro. Since its introduction in the 1950s its use has been established world-wide, irrespective of evident limitations regarding scientific as well as ethical points of view ${ }^{8}$. Fetal serum is basically a by-product of meat production collected from the still beating heart of living fetuses. While efforts are made to reduce the use of FCS, they show rather limited successes ${ }^{9}$.

As for other supplements of natural origin, the main variability source associated to FBS can be traced back to largely uncharacterized bioactive components. Due to low concentrations or lack of experimental standard measurements, they may remain poorly controlled, but may still influence the outcome of cell-based experiments. While some effort has been spent to define the composition of FCS, the main bioactive constituents subjected to meaningful variation are hardly known ${ }^{8}$. Batchdependent variations have been described to affect biological outcomes but such considerations remain limited to rather specialized topics such as hormone regulation ${ }^{10}$. Chemically defined media (CDM) represent a general and consequent solution for these problems, but are only established and available for a limited number of cell model systems ${ }^{9}$.

The focus of the present study was to investigate whether it was possible to identify bioactive compounds in FCS accounting for relevant batch-specific effects and correlate this information with proven and biochemically evident readouts on cell functions. Variations of amino acid and metabolite composition of FCS appeared less likely as it should be limited due to the homoeostatic control of the organism and, further limited by the dilution of FCS in the cell culture media (typically 5-10\%). Thus proteins as well as eicosanoids and other polyunsaturated fatty acids (PUFAs) profiled as the most relevant bioactive candidate molecules to account for inter-batch variation. Indeed, they are responsible of regulating biological processes associated with inflammation ${ }^{11}$ and inflammationassociated pathomechanisms $\mathrm{s}^{12,13,14}$. The monocyte cell line U937, a well-established cell model for 
macrophages ${ }^{15,16,17}$, was chosen for these investigations. Overall, the data demonstrated significant effects of FCS-contained eicosanoids with batch-dependent variations on relevant cell functions, proving that bioactive lipids content in serum contributes to reproducibility issues in cell culture experiments.

\section{Results}

Formation of bioactive pro-inflammatory mediators by macrophages may be influenced by FCS batch effects

In order to systematically investigate cell culture reproducibility issues resulting from FCS batch effects, a proteome profiling experiment using a U937 macrophage differentiation and activation model was performed. A single batch of U937 cells was seeded into 24 identical aliquots, forming 4 groups subsequently sub-cultured with four different FCS batches (Table 1, see Materials and Methods). All cells were differentiated using PMA to induce macrophage formation as verified by FACS analysis (Supplementary Figure S1), while three aliquots of each group (FCS batch) were subsequently treated with LPS to induce inflammatory stimulation, the other three per group serving as untreated controls. The formation of inflammatory mediators was investigated by comparative secretome analysis resulting in the identification of 488 proteins (Supplementary Table S3) and 54 eicosanoids and fatty acid precursor molecules (Supplementary Table S4). Whereas most molecules such as the chemokine CCL3 and CXCL5 showed rather little variation between the groups, reproducibility issues of differentiated U937 macrophages were evidenced by FCS batch-dependent significant $(F D R<0.05)$ differences in the formation of the chemokine $C C L 5$, the cell growth regulator IGFBP2, and the cell migration and fibrinolysis regulator SERPINE1 (PAI1) and MMP1 (Figure 1A). In line, the amount of bioactive eicosanoids comprising the hydroxyeicosatetraenoic acids 11-, 12-, and 15-HETE, hydroxydocosahexaenoic acid 17-HDoHE, the prostaglandin PGJ2 and other were found to differ significantly (FDR<0.05) depending on the FCS batch used for cell culture (Figure 1B).

Inflammatory stimulation with LPS induced the secretion of a total of 67 proteins (FDR $<0.05$, Supplementary Table S3), including tumor necrosis factors TNF and TNFSF15, chemokines such as CCL3, CXCL5, CXCL10, metalloproteinases including MMP1 and MMP10 and other promoters and mediators of inflammation (Figure $1 \mathrm{~A}$ and Supplementary Table S3). Only 22 of those 67 proteins were found uniformly regulated independent of the FCS batch (Figure 1C), whereas other bioactive molecules such as IGFBP2 and TNFSF15 again showed FCS batch-dependent expression patterns (Figure 1C). Similarly, LPS treatment induced the formation of lipid mediators of inflammation such as 15-HETE, PGE2, PGJ2 and others (Figure 1B, Supplementary Table S4). The formation of 
eicosanoids varied rather strongly dependent on the FCS batch used, four out of seven LPS-induced eicosanoids showing significant batch-dependent alterations (FDR<0.05, Figure 1D).

The eicosanoid content of FCS varies in a batch-dependent fashion

The induction of inflammatory activities of cells may be subject to modulation by a delicate balance of pro- and anti-inflammatory molecules. Thus we investigated whether the above-described batch effects may be caused by differences of the protein and eicosanoid content of the FCS batches used for the cell culture experiments. Remarkably, the protein profile comprising 289 identified proteins $(F D R<0.01)$ of the four different FCS batches was rather consistent (Figure 2A, Supplementary Table S5). Several significant abundance differences between batches were observed (Supplementary Table S5), and a principle component analysis fairly well clustered the FCS samples according to batches (Figure 2B). The analysis of FCS eicosanoid contents revealed even stronger batchdependent differences, comprising mainly COX and LOX-products (Figure 2C, Supplementary Table S6). Here, an unbiased PCA clustered the FCS batches with clear distances between batch clusters (Figure 2D), and demonstrated that batch dependent variation of eicosanoid content exceeded the variation of protein content in FCS.

\section{Cell culture is subjecting fatty acids to a high turnover}

Growing cells require medium supply of fatty acids and fetal calf serum is rich in polyunsaturated fatty acids. In order to mediate biological effects as assumed for the FCS-contained fatty acids described above (Figure 2B), cells are supposed to take up fatty acids from the medium. In order to estimate to what extent cultured $U 937$ cells may be able to take up fatty acids from medium and release fatty acids back into medium upon stimulation, we investigated the intracellular to extracellular exchange dynamics of the eicosanoid precursor molecule arachidonic acid (AA). For this purpose, cultured U937 cells were spiked with stable isotope labelled AA at a concentration double of the endogenous AA (1.6 $\mu \mathrm{M})$. Stable isotope labelled AA can be clearly distinguished from endogenous AA by mass spectrometry. As demonstrated in Figure 3, upon differentiation to macrophages, U937 cells apparently picked up more than $99 \%$ of the labelled and spiked AA within 72 hours and less than 1\% C13-AA remained detectable in the medium after that period. As expected, subsequent LPS treatment triggered a significant increase of the amount of C13-AA in the supernatant (Figure 3). This demonstrated that phospholipase activity was capable of releasing previously incorporated AA back into the medium. When supplementation with C13-AA was 
performed after PMA differentiation but before LPS treatment, the outcome was similar. Around $95 \%$ of the available AA was incorporated, but still a significant release of C13-AA was observed upon LPS treatment, clearly indicating a high turnover rate of AA. Concomitant measurement of endogenous C12-AA confirmed that AA was consumed substantially during cell culture and released back in the medium again to a smaller proportion upon LPS treatment.

Supplementation of 5-HETE and 15-HETE in the nanomolar concentration range induces the formation of peroxisomes in U937 macrophages

In order to demonstrate that the detected differences in eicosanoid content of FCS could originate from the observed batch effects, we performed an additional proteome profiling experiment with U937 cells at conditions only differing with regard to two selected eicosanoids, 5-HETE and 15-HETE. To this aim, we supplemented the FCS batch found to have lowest levels of these two eicosanoids, here designated FCS-B (containing $5 \mathrm{nM} \mathrm{5-HETE}$ and $12 \mathrm{nM}$ 15-HETE), with the pure chemicals to levels close to those observed in case of FCS batch A (FCS-A, containing 42 nM 5-HETE and 49 nM 15HETE, Figure 4A). Thus, U937 cells were grown and differentiated as before, using either FCS-A, supplemented FCS-A, or FCS-B, and subsequently subjected to proteome profiling of cytoplasmic proteins (Figure 4B). Indeed, spiking in of the two HETEs was associated with distinct proteome alterations (Figure 4B) including down-regulation of PKM and up-regulation of PEX 16, a peroxisomal membrane biogenesis protei ${ }^{18}$. To independently verify with a complementary method that this was a relevant observation, peroxisome formation was analysed using immunofluorescence with an anti-PMP70 antibody. Nuclei and mitochondria were additionally stained to demonstrate uniform appearance of these organelles serving as background control. Indeed, treatment of U937 cells with increasing concentration of HETEs induced the formation of peroxisomes in an apparently concentration dependent manner (Figure 4C).

\section{Discussion}

The present data demonstrates that variations in the eicosanoid content of FCS may account for substantial batch effects with regard to functional readouts of a cell culture model reporting inflammatory mediators. This finding may be of great relevance for a large number of laboratories working with cell culture and using FCS, as FCS-contained eicosanoids have hardly been considered to have major implications for cell culture experiments and have thus, to the best of our knowledge, not yet been subjected to rigorous control. There are reasons, why relevant effects of eicosanoids 
bioRxiv preprint doi: https://doi.org/10.1101/2020.09.18.303313; this version posted September 18, 2020. The copyright holder for this preprint (which was not certified by peer review) is the author/funder, who has granted bioRxiv a license to display the preprint in perpetuity. It is made available under aCC-BY-ND 4.0 International license.

contained in FCS were hardly expected. First of all, these molecules are generally considered to be short-lived and to act mainly in situ ${ }^{19}$. Secondly, eicosanoids were detected in FCS in the lower nanomolar concentration range, this is much less than the concentration range applied for functional assays in vitro, which is typically around $1 \mu \mathrm{M}^{20,21}$. Furthermore, fatty acids including eicosanoids contained in serum are bound to albumin and only about $0.1 \%$ is actually free from associated molecules $22,23,24$. This free pool has a high turnover rate of about 2 min accounting for the redistribution of albumin-bound fatty acids in vivo to distant organs such as muscles or the liver.

When investigating FCS batch effects, we initially expected proteins to represent the most plausible candidates contributing to reproducibility issues. Proteins as well as metabolites are strictly regulated in vivo to ensure homeostasis and consequently stable viability of the organism. While proteins may be rather stable in biological environments, metabolites such as fatty acids are much more vulnerable to chemical reactions such as oxidation, which may occur also during processing of FCS and are hard to control. Thus it was somewhat unexpected to see that FCS eicosanoid profiles were stable and clustered the FCS samples according to batches (Figure 2). This finding, supported by older and current literature reporting remarkable biological effects of eicosanoids ${ }^{25,26,27}$, motivated us to focus on this class of molecules. Functional analyses were to be based on spiking experiments with the U937 cells. As a first step, the efficient and fast uptake of albumin bound arachidonic acid (AA) was verified in the present cell model system using stable isotope labelled AA. The subsequent release of labelled AA upon LPS stimulation of the cells strongly indicated the previous uptake and incorporation of AA into more complex lipids, from where AA was apparently released by the action of LPS-induced phospholipase $A 2{ }^{28}$. In order to test potential biological effects of eicosanoids on U937 cells, a decision was made in favour of commercially available eicosanoids, 5-HETE and 15-HETE, which were found to show remarkable concentration differences between the FCS batches. Hydroxyeicosatetraenoic acids (HETEs) are formed with AA by the action of lipoxygenases ALOX5 and ALOX15, expressed typically by epithelial cells as well as phagocytes such as neutrophils and macrophages ${ }^{29,30}$. Beside their effects on cell proliferation and differentiation, they are known activators of PPAR ${ }^{31}$. Actually, peroxisome proliferator-activated receptors are known to induce the uptake and metabolism of fatty acids and to strongly modulate immune functions ${ }^{32}$. As fatty acid metabolism takes place in peroxisomes ${ }^{33}$, the 5 - HETE/15-HETE induced up-regulation of PEX16 (Figure 4), a peroxisome biogenesis protein indicative for peroxisome proliferation ${ }^{18}$, indicated that this treatment caused an increased demand for these organelles. The concomitant down-regulation of PKM (Figure 4), a key enzyme for glycolysis ${ }^{34}$, may suggest that HETE-treatment of U937 cells induced a metabolic shift increasing beta oxidation and attenuating glycolysis. This interpretation was independently supported by the concentration- 
dependent HETE-induced formation of peroxisomes (Figure 4) observed by immunofluorescence staining using a PMP70 antibody ${ }^{35}$.

\section{Conclusion and outlook}

The present data demonstrate that batch-dependent differences of eicosanoids contained in FCS may have a profound effect on cellular functions as observed with the U937 in vitro cell culture model for differentiation and inflammatory stimulation. Eicosanoids affect many relevant cellular events far beyond that, suggesting that they may represent main contributors for reproducibility issues in cell culture. The establishment of a strict quality control regime controlling eicosanoid content in FCS may alleviate this challenging problem.

\section{Materials and Methods}

\section{Cell Culture}

U937 cell line was cultured in RPMI medium (1X with L-Glutamine; Gibco, Thermo Fischer Scientific, Austria) supplemented with $1 \%$ Penicillin/Streptomycin (Sigma-Aldrich, Austria) and 10\% Fetal Calf Serum (FCS, Sigma-Aldrich, Austria) in T25 polystyrene cell culture flasks for suspension cells (Sarstedt, Austria) at $37{ }^{\circ} \mathrm{C}$ and $5 \% \mathrm{CO}_{2}$. Cells were counted with a MOXI Z Mini Automated Cell Counter (ORFLO Technologies, USA) using Moxi Z Type M Cassettes (ORFLO Technologies, USA) and the number of seeded cells for the experiments calculated based of these results. For all experiments the cells were used in passages 22-26.

Differentiation with Phorbol 12-myristate 13-acetate (PMA) and inflammatory activation with Lipopolysaccharides (LPS):

All experiments were carried out in triplicates of LPS activation and control. For the proteomics and eicosadomics measurements $2 \times 10^{\wedge} 6$ cells were seeded in T25 polystyrene cell culture flasks with cell growth surface for adherent cells (Sarstedt, Austria) with $5 \mathrm{ml}$ fully supplemented media and 100 $\mathrm{ng} / \mathrm{ml}$ PMA (Phorbol 12-myristate 13 -acetate $\geq 99 \%$, Sigma-Aldrich, Austria) to induce differentiation. After $48 \mathrm{~h}$ incubation the medium was withdrawn and used for eicosanoid measurements. $3 \mathrm{ml}$ of new fully supplemented media was added either with $1 \mu \mathrm{g} / \mathrm{ml}$ LPS (Lipopolysaccharides from Escherichia coli 055:B5, $\gamma$-irradiated, BioXtra, Sigma-Aldrich, Austria) or $1 \mu \mathrm{l}$ PBS per $1 \mathrm{ml}$ medium as 
control. After $24 \mathrm{~h}$ activation the medium was withdrawn again and used for eicosanoid measurements. The cells were gently washed twice with $5 \mathrm{ml}$ PBS and $3 \mathrm{ml}$ new medium without FCS was added and incubated. After 4 hours the supernatant was withdrawn and used for proteomics measurements. The cells were used for a subcellular fractionation as described before and cytoplasm and nuclear fraction were used for proteomics analysis ${ }^{36}$.

\section{Test of different FCS batches}

Throughout the experiments different suppliers and batches of FCS have been used. Additional details concerning the FCS batches are listed in table 1. FCS batches A-C were heat inactivated at $56^{\circ} \mathrm{C}$ for $30 \mathrm{~min}$, batch $\mathrm{D}$ was already bought heat inactivated. Also different concentrations of HETES and labelled Arachidonic acid have been supplemented and the respective controls have been treated with the same amount of LC-MS grade methanol $(5 \mu \mathrm{l} / 3 \mathrm{ml}$ medium). The experimental workflow of PMA differentiation and LPS activation was done for every condition similarly, only exchanging the FCS batch, supplier or eicosanoid. Additionally, for every condition 3 aliquots $(3 \times 3 \mathrm{ml})$ of the fully supplemented media were used for eicosanoid measurements to determine the default levels of eicosanoids present.

\begin{tabular}{|c|c|c|c|c|c|}
\hline Nomenclature & Vendor & Lot number & Expiration date & Origin & Processed \\
\hline A & Sigma & BCBT4187 & 07.2021 & & - \\
\hline B & Gibco & 42 Q5650K & 06.2020 & Brazil & - \\
\hline C & Gibco & 42 G8378K & 11.2022 & Brazil & - \\
\hline D & Gibco & 08 Q8082K & 02.2023 & Brazil & Heat inactivated \\
\hline
\end{tabular}

Table 1: Tested FCS batches stating Vender, Lot number, expiration date as well as letter used in this work.

\section{C13 labelled arachidonic acid}

For the investigation of the uptake and release of PUFAs an experiment was carried out with the supplementation of $1,2,3,4,5-{ }^{13} \mathrm{C}$ arachidonic acid (C13 AA, Cayman chemicals, USA). Whenever C13 AA was added, a control experiment was supplemented with the same concentration of unlabelled arachidonic acid. For the first experiment the $\mathrm{C} 13$ AA was added at a concentration of $1.6 \mu \mathrm{M}$ (used for all AA supplementations) to the fully supplemented medium during the 48h PMA differentiation step. This concentration is around double of the endogenous arachidonic acid, thus the supplementation tripled the concentration of biologically active arachidonic acid. Afterwards, the now adherent cells were washed three time with PBS and medium without supplemented AA was 
added together with or without LPS for $24 \mathrm{~h}$. For the second experiment the cells were differentiated with PMA in standard medium, washed three times with PBS and medium supplemented with C13 AA was added together with and without LPS for $24 \mathrm{~h}$. The eicosanoids were collected and measured in the supplemented medium without incubation (t0), after $48 \mathrm{~h}$ PMA differentiation and after $24 \mathrm{~h}$ LPS activation. The experimental setup is illustrated in Figure 3.

Proteomics of supernatant (SN), cytoplasm (CYT) and nuclear extract (NE)

For the proteomics sample preparation, the s-trap system (Protifi, Huntington, NY) was employed following the manufacturers protocol with slight modifications. The precipitated proteins were dissolved in lysis buffer (8 M Urea, $0.05 \mathrm{M}$ TEAB and 5\% SDS) and diluted to get a protein concentration of about $1 \mu \mathrm{g} / \mu \mathrm{l} .20 \mu \mathrm{g}$ of protein were used for each digestion. First, the sample was reduced with $20 \mu \mathrm{L}$ DTT (Sigma-Aldrich) at a final concentration of $32 \mathrm{mM}$ for $10 \mathrm{~min}$ at $95{ }^{\circ} \mathrm{C}$. Afterwards, $5 \mu$ I IAA (Sigma-Aldrich) was added to a final concentration of $54 \mathrm{mM}$ and incubated for $30 \mathrm{~min}$ at $30^{\circ} \mathrm{C}$ in the dark. After adding 4,5 $\mu \mathrm{l}$ 12\% ortho-phosphoric acid (Sigma-Aldrich) and $297 \mu \mathrm{l}$ S-Trap buffer ( $90 \%$ Methanol $(\mathrm{v} / \mathrm{v})$ in $\mathrm{H}_{2} \mathrm{O}$ and $0.1 \mathrm{M}$ TEAB) the sample was loaded onto the S-Trap Filter. The S-trap filters were centrifuged at $4000 \mathrm{~g}$ for $1 \mathrm{~min}$ to pass through all sample and trap the proteins onto the resin and afterwards washed four times with $150 \mu \mathrm{l} \mathrm{S-Trap} \mathrm{buffer.} 20 \mu \mathrm{g}$ aliquots of Trypsin/Lys-C (MS grade; Promega Corporation, Madison, WI, USA) were dissolved in $400 \mu \mathrm{I} 50$ $\mathrm{mM} \mathrm{TEAB}$ and $20 \mu \mathrm{g}$ of this solution was added directly onto the resin of the filter (corresponding to $1 \mu \mathrm{g}$ Trypsin/Lys-C per sample) and incubated for $1 \mathrm{~h}$ at $47{ }^{\circ} \mathrm{C}$. After finishing the digestion, the peptides were eluted with $40 \mu \mathrm{l}$ of $50 \mathrm{mM}$ TEAB followed by $40 \mu \mathrm{l}$ of $0.2 \%$ formic acid (FA) in $\mathrm{H}_{2} \mathrm{O}$ and $35 \mu \mathrm{l}$ of $50 \%(\mathrm{v} / \mathrm{v})$ acetonitrile (ACN) with $0.2 \% \mathrm{FA}$ in $\mathrm{H}_{2} \mathrm{O}$. The peptides were dried for about 2 $\mathrm{h}$ via vacuum centrifugation and stored at $-20^{\circ} \mathrm{C}$ until LC-MS/MS measurement.

\section{HPLC-MS/MS for proteomics}

For the HPLC-MS/MS analysis the peptides were resolved in $5 \mu \mathrm{L} 30 \%$ formic acid and diluted with 40 $\mu \mathrm{L}$ of mobile phase $\mathrm{A}\left(97.9 \% \mathrm{H}_{2} \mathrm{O}, 2 \%\right.$ acetonitrile, $0.1 \%$ formic acid). $1 \mu \mathrm{L}$ for the supernatant samples and $5 \mu \mathrm{L}$ of cytoplasmic and nuclear samples were injected into the Dionex UltiMate 3000 RSLCnano LC system coupled to the QExactive Orbitrap MS (all Thermo Fisher Scientific, Austria). Peptides were trapped on a C18 $2 \mathrm{~cm} \times 100 \mu \mathrm{m}$ precolumn and LC separation was performed on a 50 $\mathrm{cm} \times 75 \mu \mathrm{m}$ Pepmap100 analytical column (both Thermo Fisher Scientific, Austria). Separation was achieved applying a 43 min gradient from $7 \%$ to $40 \%$ mobile phase $B\left(79.9 \%\right.$ acetonitrile, $20 \% \mathrm{H}_{2} \mathrm{O}$, 
$0.1 \%$ formic acid) for supernatant samples and 95 min gradients from $8 \%$ to $40 \%$ mobile phase $B$ for cytoplasmic and nuclear samples, both at a flow rate of $300 \mathrm{~nL} / \mathrm{min}$, resulting in a total run time of $85 \mathrm{~min}$ and $135 \mathrm{~min}$, respectively. Mass spectrometric settings were the same for all fractions. The resolution on the MS1 level was set to $70,000($ at $m / z=200)$ with a scan range from 400 to 1,400 $\mathrm{m} / \mathrm{z}$. The top eight abundant peptide ions were chosen for fragmentation at $30 \%$ normalized collision energy and resulting fragments analyzed in the Orbitrap at a resolution of $17,500($ at $m / z=$ 200).

\section{Proteomics Data Analysis}

Raw data were subjected to the freely available software MaxQuant (version 1.6.0.1) ) $^{37}$ utilizing the Andromeda search engine, followed by statistical evaluation with the Perseus software (version 1.6.0.2) ${ }^{38}$. For the MaxQuant search, a minimum of two peptide identifications, at least one of them being a unique peptide, was required for valid protein identification. Digestion mode was set to "Specific" choosing Trypsin/P. The peptide mass tolerance was set to $50 \mathrm{ppm}$ for the first search and to $25 \mathrm{ppm}$ for the main search. The false discovery rate (FDR) was set to 0.01 both on peptide and protein level, based on the q-value. The database applied for the search was the human Uniprot database (version 03/2018, reviewed entries only) with 20,316 protein entries. Further settings for the search included carbamidomethylation as fixed modification and oxidation of methionine and acetylation of the protein $C$ terminus as variable modifications. Each peptide was allowed to have a maximum of two missed cleavages and two modifications, "Match between runs" was enabled and the alignment window set to $10 \mathrm{~min}$, with the match time window of $1 \mathrm{~min}$. The mass spectrometry proteomics data have been deposited to the ProteomeXchange Consortium (http://proteomecentral.proteomexchange.org) via the PRIDE partner repository ${ }^{39}$ with the dataset identifier PXD020617 and 10.6019/PXD020617.

\section{Eicosanoid sample preparation}

Cell supernatants were spiked with $5 \mu \mathrm{l}$ of internal standards (Supplementary Table S1) and centrifuged at $726 \mathrm{~g}$ for $5 \mathrm{~min}$ to remove cells and debris. $3 \mathrm{ml}$ of that supernatant was mixed with $12 \mathrm{ml}$ of ice cold ethanol and left at $-20^{\circ} \mathrm{C}$ over night to precipitate the contained proteins. The samples were centrifuged for $30 \mathrm{~min}$ with $4536 \mathrm{~g}$ at $4{ }^{\circ} \mathrm{C}$ and the supernatant transferred into a new $15 \mathrm{ml}$ Falcon tube. Ethanol was evaporated via vacuum centrifugation at $37^{\circ} \mathrm{C}$ until the original sample volume was restored. Samples were loaded on conditioned $30 \mathrm{mg} / \mathrm{ml} \mathrm{StrataX}$ solid phase 
extraction (SPE) columns (Phenomenex, Torrance, CA, U.S.A.). Columns were washed with $2 \mathrm{ml} \mathrm{MS}$ grade water and eicosanoids were eluted with $500 \mu \mathrm{L}$ methanol (MeOH abs.; VWR International, Vienna, Austria) containing 2\% formic acid (FA; Sigma-Aldrich). $\mathrm{MeOH}$ was evaporated using $\mathrm{N}_{2}$ stream at room temperature and reconstituted in $150 \mu \mathrm{L}$ reconstitution buffer $(\mathrm{H} 2 \mathrm{O} / \mathrm{ACN} / \mathrm{MeOH}+$ $0,2 \%$ FA - 65:31,5:3,5), containing a second set of internal eicosanoid standards at a concentration of 10-100 nM (Supplementary Table S1).

UHPLC-MS/MS for eicosanoid measurements

Analytes were separated using a Thermo Scientific Vanquish (UHPLC) system and a Kinetex ${ }^{\circledR}$ C18 -

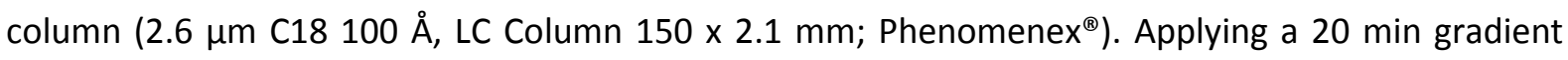
flow method, starting at 35\% B steadily increasing to $90 \%$ B (1-10min), going up to $99 \%$ B in 0.25 min. Flow rate was kept at $200 \mu \mathrm{L} / \mathrm{min}, 20 \mu \mathrm{L}$ injection volume and column oven temperature was set to $40^{\circ} \mathrm{C}$. Eluent A contains $\mathrm{H} 2 \mathrm{O}+0.2 \% \mathrm{FA}$ and eluent B ACN:MeOH $(90: 10)+0.2 \% \mathrm{FA}$.

Mass Spectrometric analysis was performed with a Q Exactive HF Quadrupole-Orbitrap mass spectrometer (Thermo Fisher Scientific, Austria), equipped with a HESI source for negative ionization. Mass spectra were recorded operating from 250 to $700 \mathrm{~m} / \mathrm{z}$ at a resolution of $60000 @$ $200 \mathrm{~m} / \mathrm{z}$ on MS1 level. The two most abundant precursor ions were selected for fragmentation (HCD 24 normalized collision energy), preferentially molecules from an inclusion list which contained 32 $\mathrm{m} / \mathrm{z}$ values specific for eicosanoids (Supplementary Table S2). MS2 was operated at a resolution of $15000 @ 200 \mathrm{~m} / \mathrm{z}$. For negative ionization, a spray voltage of $2.2 \mathrm{kV}$ and a capillary temperature of $253^{\circ} \mathrm{C}$ were applied, with the sheath gas set to 46 and the auxiliary gas to 10 arbitrary units.

Generated raw files were analyzed manually using Thermo Xcalibur 4.1.31.9 (Qual browser), comparing reference spectra from the Lipid Maps depository library from July $2018^{40}$. For peak integration the software TraceFinder $^{\mathrm{TM}}$ (version 4.1 - Thermo Scientific, Austria) was used.

\section{Immunofluorescence}

For fluorescence microscopy $8 * 10^{4}$ cells in $400 \mu \mathrm{L}$ were seeded in a $\mu$-Slide 8 well (Ibitreat coating, ibidi GmbH Martinsried, Germany). Differentiation of the cells was induced with $100 \mathrm{ng} / \mathrm{ml} \mathrm{PMA} \mathrm{for}$ $48 \mathrm{~h}$. Afterwards the cell supernatant was exchanged with fully supplemented medium without PMA for additional $24 \mathrm{~h}$. Sample preparation was performed as previously described with minor modifications $^{41}$. Cells were fixed with pre-warmed formaldehyde (3.7\%) for $15 \mathrm{~min}$ and permeabilised with Triton-X $100(0.2 \%)$ for 10 min. Blocking was performed with Donkey serum (2\% 
in PBS-A) for $1 \mathrm{~h}, \mathrm{RT}$. Primary antibodies were incubated $2 \mathrm{~h}$ at RT at dilution 1:500. After washing, specie-specific fluorescent-labelled secondary antibodies were added and slides incubated in a dark humidified chamber for $1.5 \mathrm{~h}$. For our study, Anti PMP70 Antibody (Rabbit polyclonal, PA1-650) and Anti TOM20 (F-10, Mouse Monoclonal Sc-17764), Alexa Fluor 488 Donkey Anti Mouse (A21202_LOT2090565) and Alexa Fluor 568 Donkey Anti-Rabbit (A10042_LOT2136776) were used. The slides were washed and post-fixed with 3.7\% formaldehyde (10 min, RT); at the end of the postfixation, $100 \mathrm{mM}$ glycine was used to mask reactive sites and slides were mounted and sealed with Roti-Mount FluoCare (Roth, Graz, Austria) with DAPI. SIM Images were acquired with a Confocal LSM Zeiss 710 equipped with ELYRA PS. 1 system. Structured Illumination Microscopy (SIM) images were obtained with (Plan Apochromat 63X/1.4 oil objective) grid 5 rotation.

\section{Differentiation status by flow cytometry}

In order to confirm the differentiation status obtained via PMA treatment the cells were tested for the differentiation marker CD11b (ITGAM) using FACS analysis. Therefore, U937 cells were treated with $100 \mathrm{ng} / \mathrm{mL}$ PMA for $48 \mathrm{~h}$ using $2 \times 105$ cells per well in 6-well plates. After the incubation time, the cells were washed three times with PBS and put on ice. The differentiation status was assessed by labelling with an anti-CD11b antibody (APC clone D12, BD Bioscience) and subsequent evaluation of the CD11b+ population. Three biological replicates were analysed per condition on a FACS Canto II cytometer (BD Bioscience).

\section{Acknowledgements}

We acknowledge support by the Mass Spectrometry Center of the Faculty of Chemistry, University of Vienna, member of the Vienna Life Science Instruments (VLSI).

\section{Additional information}

Supplementary information 


\section{References}

1. Prinz F, Schlange T, Asadullah K. Believe it or not: how much can we rely on published data on potential drug targets? Nat Rev Drug Discov 10, 712 (2011).

2. Baker M. Reproducibility: Respect your cells! Nature 537, 433-435 (2016).

3. Wolfensohn S. A review of the contributions of cross-discipline collaborative European IMI/EFPIA research projects to the development of Replacement, Reduction and Refinement strategies. Altern Lab Anim 46, 91-102 (2018).

4. Del Favero G, Kraegeloh A. Integrating Biophysics in Toxicology. Cells 9, (2020).

5. Freedman LP, Cockburn IM, Simcoe TS. The Economics of Reproducibility in Preclinical Research. PLoS Biol 13, e1002165 (2015).

6. Ben-David $U$, et al. Genetic and transcriptional evolution alters cancer cell line drug response. Nature 560, 325-330 (2018).

7. Liu Y, et al. Multi-omic measurements of heterogeneity in HeLa cells across laboratories. Nat Biotechnol 37, 314-322 (2019).

8. Gstraunthaler G, Lindl T, van der Valk J. A plea to reduce or replace fetal bovine serum in cell culture media. Cytotechnology 65, 791-793 (2013).

9. van der Valk J, et al. Fetal Bovine Serum (FBS): Past - Present - Future. ALTEX 35, 99-118 (2018).

10. Sikora MJ, Johnson MD, Lee AV, Oesterreich S. Endocrine Response Phenotypes Are Altered by Charcoal-Stripped Serum Variability. Endocrinology 157, 3760-3766 (2016).

11. Dennis EA, Norris PC. Eicosanoid storm in infection and inflammation. Nat Rev Immunol 15, 511-523 (2015).

12. Tahir A, et al. Combined Proteome and Eicosanoid Profiling Approach for Revealing Implications of Human Fibroblasts in Chronic Inflammation. Anal Chem 89, 1945-1954 (2017).

13. Reichl B, et al. Determination of a Tumor-Promoting Microenvironment in Recurrent Medulloblastoma: A Multi-Omics Study of Cerebrospinal Fluid. Cancers (Basel) 12, (2020).

14. Muqaku B, et al. Neutrophil Extracellular Trap Formation Correlates with Favorable Overall Survival in High Grade Ovarian Cancer. Cancers (Basel) 12, (2020). 
15. Sundstrom C, Nilsson K. Establishment and characterization of a human histiocytic lymphoma cell line (U-937). Int J Cancer 17, 565-577 (1976).

16. Haque MA, Jantan I, Harikrishnan H. Zerumbone suppresses the activation of inflammatory mediators in LPS-stimulated U937 macrophages through MyD88-dependent NFkappaB/MAPK/PI3K-Akt signaling pathways. Int Immunopharmacol 55, 312-322 (2018).

17. Tian X, Xie G, Ding F, Zhou X. LPS-induced MMP-9 expression is mediated through the MAPKs-AP-1 dependent mechanism in BEAS-2B and U937 cells. Exp Lung Res 44, 217-225 (2018).

18. Kim PK, Mullen RT, Schumann U, Lippincott-Schwartz J. The origin and maintenance of mammalian peroxisomes involves a de novo PEX16-dependent pathway from the ER. I Cell Biol 173, 521-532 (2006).

19. Yao C, Narumiya S. Prostaglandin-cytokine crosstalk in chronic inflammation. Br J Pharmacol 176, 337-354 (2019).

20. Zhang L, et al. TGF-beta1/FGF-2 signaling mediates the 15-HETE-induced differentiation of adventitial fibroblasts into myofibroblasts. Lipids Health Dis 15, 2 (2016).

21. Zhang L, et al. Platelet-derived growth factor (PDGF) induces pulmonary vascular remodeling through 15-LO/15-HETE pathway under hypoxic condition. Cell Signal 24, 1931-1939 (2012).

22. van der Vusse GJ. Albumin as fatty acid transporter. Drug Metab Pharmacokinet 24, 300-307 (2009).

23. Saifer A, Goldman L. Free Fatty Acids Bound to Human Serum Albumin. J Lipid Res 2, 268-\& (1961).

24. Vorum H, Pedersen AO, Honore B. Fatty-Acid and Drug-Binding to a Low-Affinity Component of Human Serum-Albumin, Purified by Affinity-Chromatography. Int J Pept Prot Res 40, 415422 (1992).

25. Umamaheswaran S, Dasari SK, Yang P, Lutgendorf SK, Sood AK. Stress, inflammation, and eicosanoids: an emerging perspective. Cancer Metastasis Rev 37, 203-211 (2018).

26. Harizi $\mathrm{H}$, Gualde N. The impact of eicosanoids on the crosstalk between innate and adaptive immunity: the key roles of dendritic cells. Tissue Antigens 65, 507-514 (2005).

27. Hammock BD, Wang W, Gilligan MM, Panigrahy D. Eicosanoids: The Overlooked Storm in Coronavirus Disease 2019 (COVID-19)? Am J Pathol 190, 1782-1788 (2020). 
28. Dieter P, Ambs P, Fitzke E, Hidaka H, Hoffmann R, Schwende H. Comparative studies of cytotoxicity and the release of TNF-alpha, nitric oxide, and eicosanoids of liver macrophages treated with lipopolysaccharide and liposome-encapsulated MTP-PE. J Immunol 155, 25952604 (1995).

29. Kuhn H, O'Donnell VB. Inflammation and immune regulation by $12 / 15$-lipoxygenases. Prog Lipid Res 45, 334-356 (2006).

30. Powell WS, Rokach J. Biochemistry, biology and chemistry of the 5-lipoxygenase product 5oxo-ETE. Prog Lipid Res 44, 154-183 (2005).

31. Powell WS, Rokach J. Biosynthesis, biological effects, and receptors of hydroxyeicosatetraenoic acids (HETEs) and oxoeicosatetraenoic acids (oxo-ETEs) derived from arachidonic acid. Biochim Biophys Acta 1851, 340-355 (2015).

32. Christofides A, Konstantinidou E, Jani C, Boussiotis VA. The role of Peroxisome ProliferatorActivated Receptors (PPAR) in immune responses. Metabolism, 154338 (2020).

33. Fransen $M$, Nordgren $M$, Wang $B$, Apanasets $O$. Role of peroxisomes in ROS/RNSmetabolism: implications for human disease. Biochim Biophys Acta 1822, 1363-1373 (2012).

34. Sato $T$, Morita $M$, Nomura $M$, Tanuma N. Revisiting glucose metabolism in cancer: lessons from a PKM knock-in model. Mol Cell Oncol 5, e1472054 (2018).

35. Zaabi NA, Kendi A, Al-Jasmi F, Takashima S, Shimozawa N, Al-Dirbashi OY. Atypical PEX16 peroxisome biogenesis disorder with mild biochemical disruptions and long survival. Brain Dev 41, 57-65 (2019).

36. Kreutz D, et al. Response Profiling Using Shotgun Proteomics Enables Global Metallodrug Mechanisms of Action To Be Established. Chemistry 23, 1881-1890 (2017).

37. Cox J, Mann M. MaxQuant enables high peptide identification rates, individualized p.p.b.range mass accuracies and proteome-wide protein quantification. Nat Biotechnol 26, 13671372 (2008).

38. Tyanova $S$, et al. The Perseus computational platform for comprehensive analysis of (prote)omics data. Nature methods 13, 731-740 (2016).

39. Vizcaino JA, et al. ProteomeXchange provides globally coordinated proteomics data submission and dissemination. Nat Biotechnol 32, 223-226 (2014).

40. Fahy E, Sud M, Cotter D, Subramaniam S. LIPID MAPS online tools for lipid research. Nucleic Acids Res 35, W606-612 (2007). 
bioRxiv preprint doi: https://doi.org/10.1101/2020.09.18.303313; this version posted September 18, 2020. The copyright holder for this preprint (which was not certified by peer review) is the author/funder, who has granted bioRxiv a license to display the preprint in perpetuity. It is made available under aCC-BY-ND 4.0 International license.

41. Del Favero G, Hohenbichler J, Mayer RM, Rychlik M, Marko D. Mycotoxin Altertoxin II Induces Lipid Peroxidation Connecting Mitochondrial Stress Response to NF-kappaB Inhibition in THP-1 Macrophages. Chem Res Toxicol 33, 492-504 (2020). 


\section{Figures and Legends:}
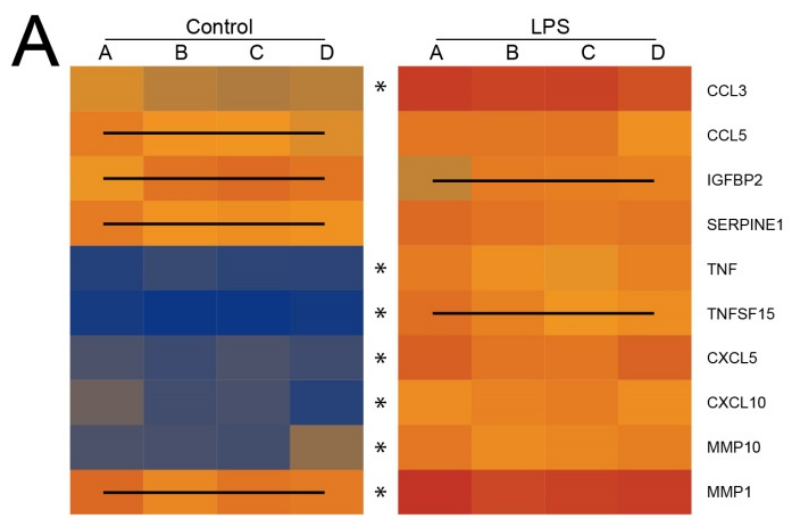

C

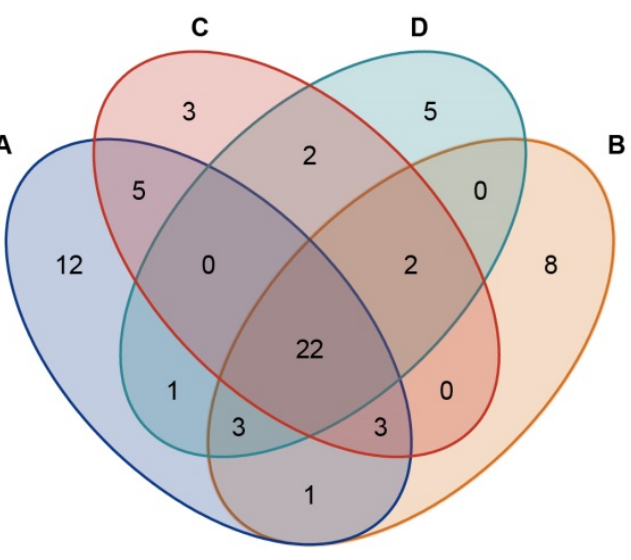

$\mathrm{B}$
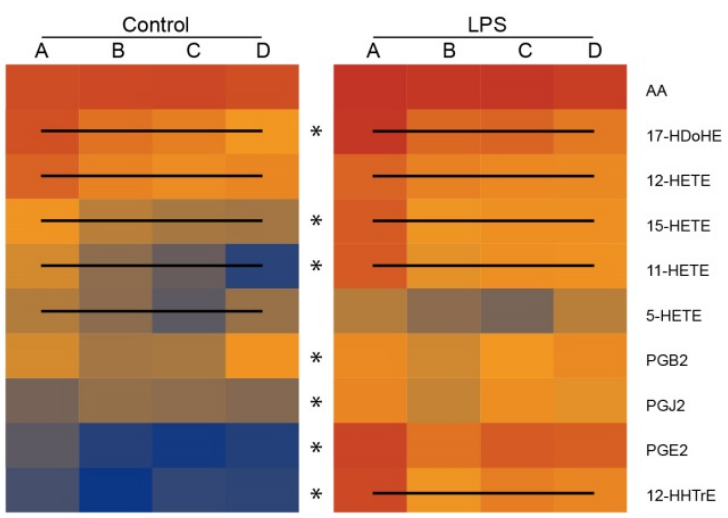

D

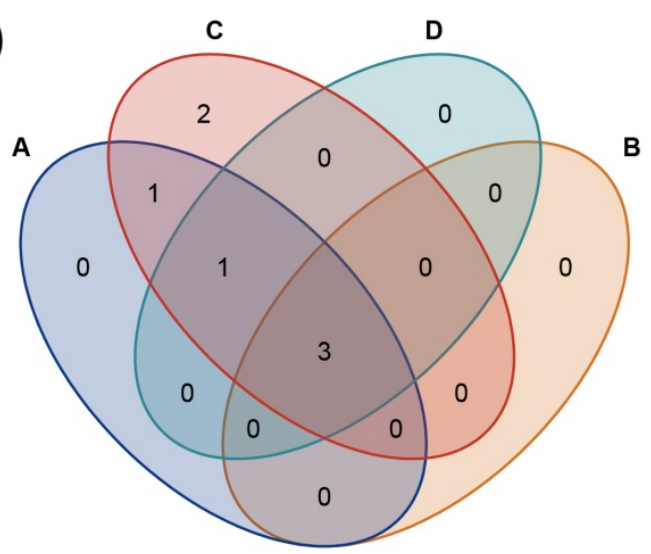

Figure 1: Heatmaps of selected proteins (A) and eicosanoids (B) determined in secretomes of control and LPS-treated U937 cells cultured with the indicated batch of FCS, A, B, C or D. Lines within a heatmap indicate a significant difference of the given molecule within at least two batches. Asterisks $\left({ }^{*}\right)$ indicate that LPS-treatment induced a significant increase. Venn diagrams of significantly up- and downregulated (C) proteins $(S 0=2, F D R=0.01)$ and (D) eicosanoids comparing LPS activation with control samples for all four FCS batches (A-D). 

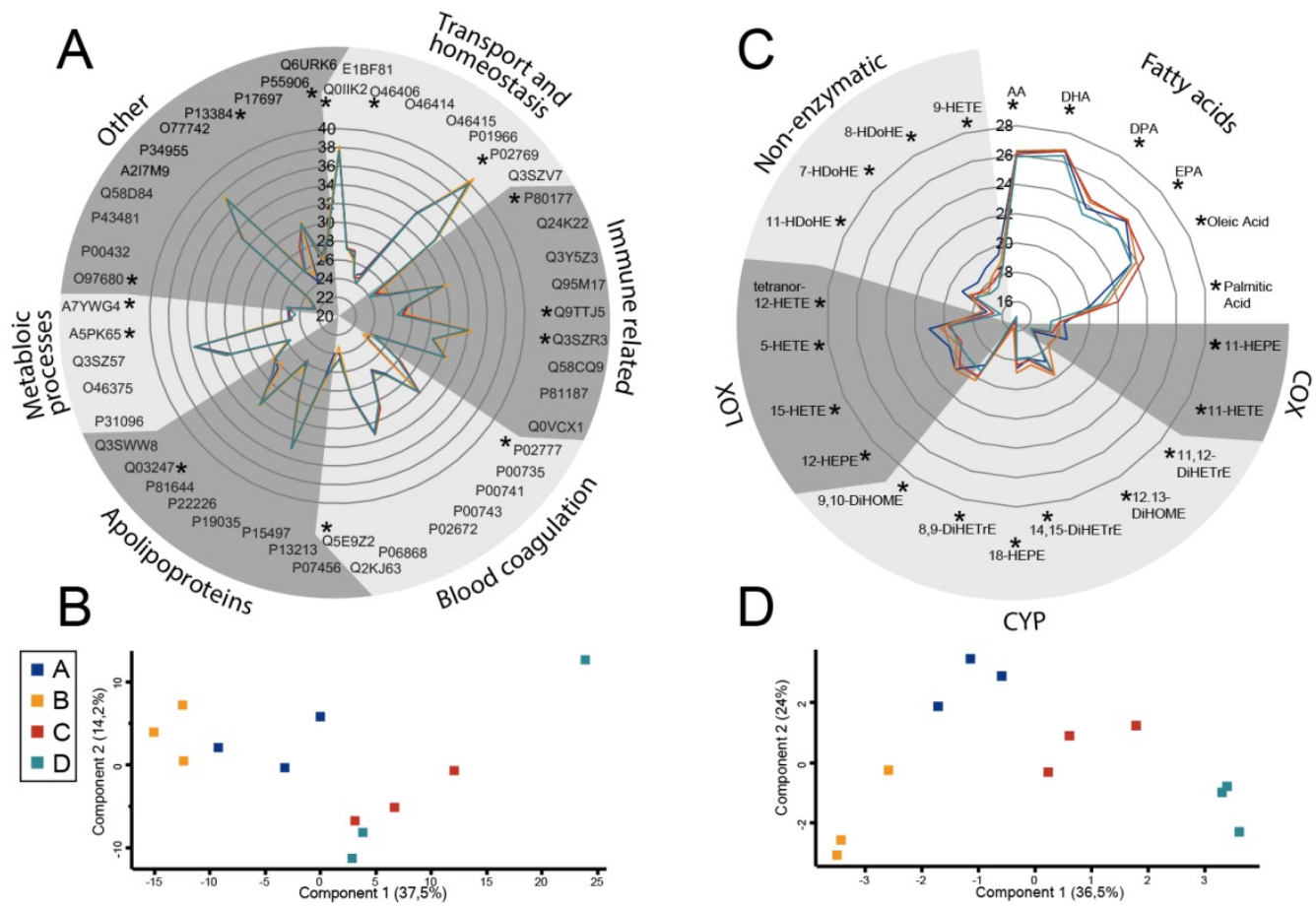

Figure 2: Radar plot for selected proteins (A) and fatty acids (C) identified in 4 different FCS batches without incubation with cells (baseline levels). Principal component analysis of protein (B) and eicosanoid (D) measurements of the same FCS batches, as indicated by different colors, demonstrates superior clustering in case of eicosanoids. 

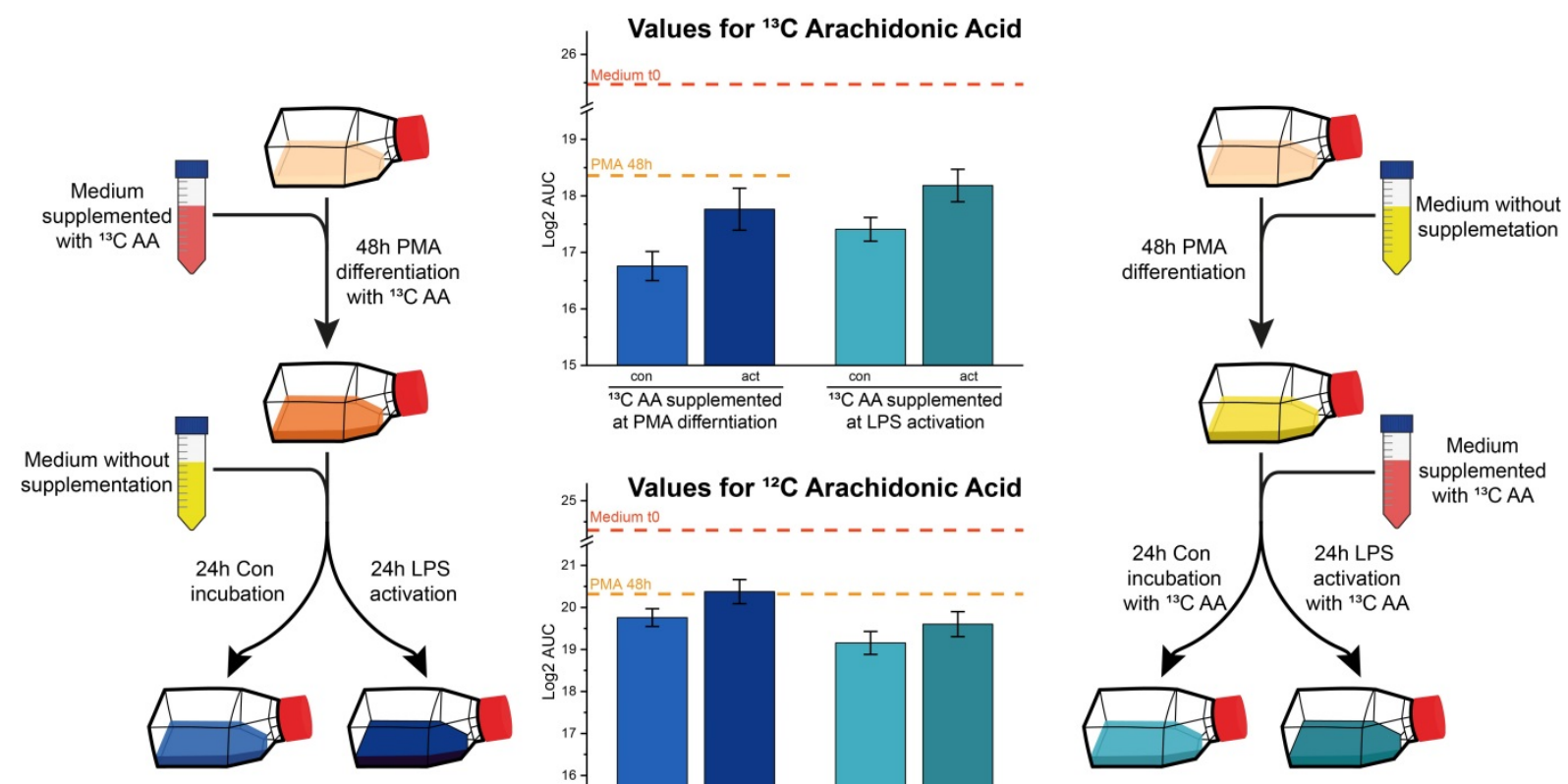

Figure 3: Experimental setup and results from AA spiking experiments. Medium was supplemented with 13C AA either before differentiation (left hand side) or before LPS treatment (right hand side). AA determination of cell supernatants by LC-MS/MS revealed AUC values as indicated. Medium levels at the beginning of cell culture are indicated by lines. Error bars are derived from three independent experiments. Con, untreated cells; act, LPS-treated cells. Note that AA concentration values strongly decrease upon cell cultivation but increase again upon LPS treatment. 

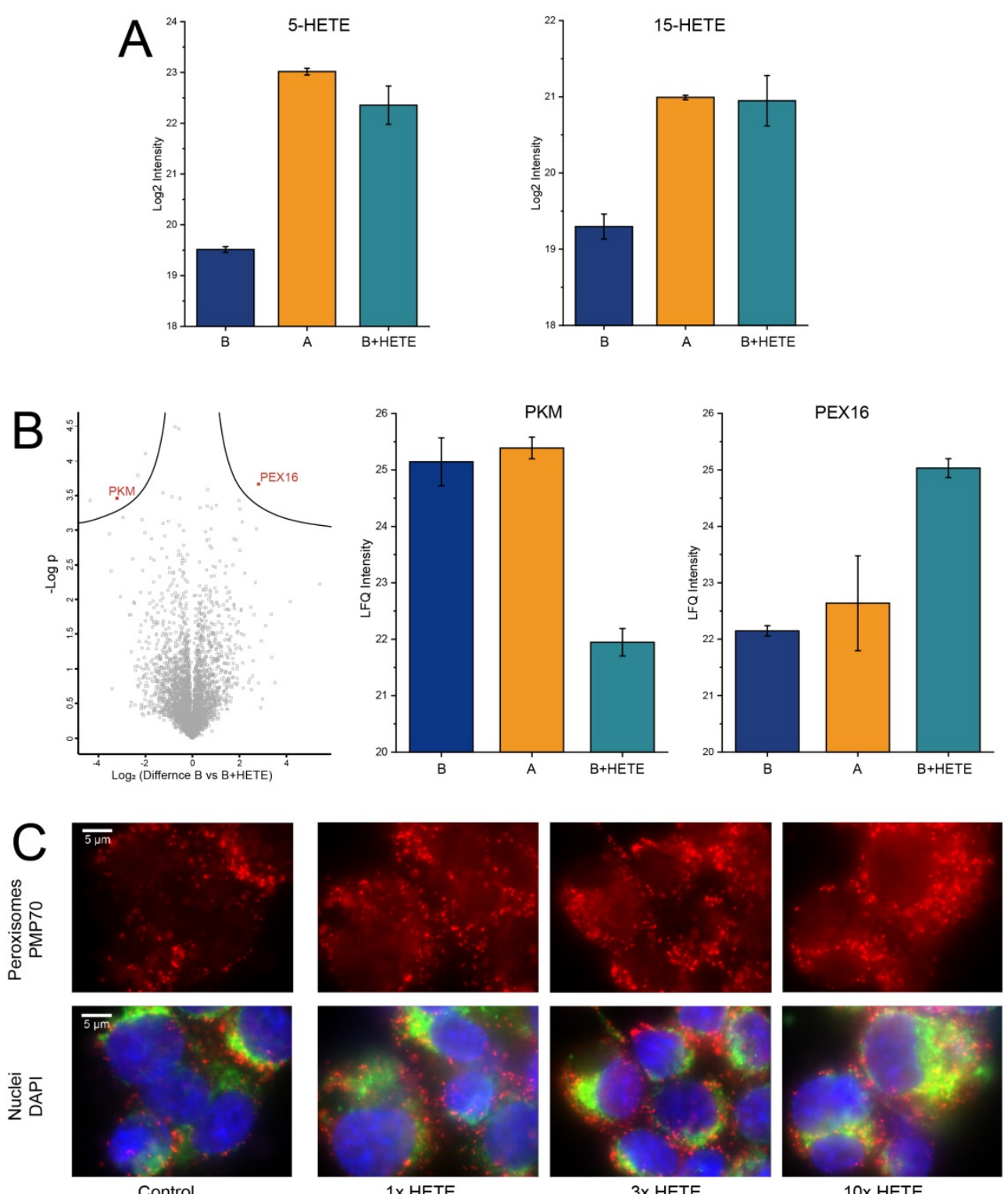

Figure 4: (A) Eicosanoid levels of 5-HETE and 15-HETE for FCS-B (before spiking), FCS-A and FCS-B after spiking with 5-HETE and 15-HETE. (B) Volcano plot for cytoplasmic proteins obtained from U937 cells after PMA differentiation when cultured in either FCS-B or FCS-B supplemented HETEs. Bar plots exemplify the significantly regulated proteins PKM and PEX16. (C) Immunofluorescence detection of peroxisomes (red, PMP70 antibody), mitochondria (green, TOM20 antibody) and nuclei (blue, DAPI) shown for control and increasing concentrations of supplemented HETEs (addition of 1 , 3 or 10 times of the spiked HETE mix). 